\title{
Stability, complex modes, and nonseparability in rotating quadratic potentials
}

\author{
R. Rossignoli and A. M. Kowalski \\ Departamento de Física-IFLP, Universidad Nacional de La Plata, C.C. 67, La Plata (1900), Argentina
}

(Received 12 December 2008; published 8 June 2009)

\begin{abstract}
We examine the dynamics of a particle in a general rotating quadratic potential, not necessarily stable or isotropic, using a general complex mode formalism. The problem is equivalent to that of a charged particle in a quadratic potential in the presence of a uniform magnetic field. It is shown that the unstable system exhibits a rich structure, with complex normal modes as well as nonstandard modes of evolution characterized by equations of motion which cannot be decoupled (nonseparable cases). It is also shown that in some unstable cases the dynamics can be stabilized by increasing the magnetic field or tuning the rotational frequency, giving rise to dynamical stability or instability windows. The evolution in general nondiagonalizable cases is as well discussed.
\end{abstract}

DOI: 10.1103/PhysRevA.79.062103

PACS number(s): 03.65.Fd, 03.65.Ca, 03.75.Kk

\section{INTRODUCTION}

Quadratic forms in boson operators or generalized coordinates and momenta are a ubiquitous presence in the theoretical description of diverse physical systems. They often arise through the linearization of the equations of motion around a stationary point, as in the case of the random-phase approximation (RPA) [1,2], providing a basic tractable scenario. They play an important role in the description of BoseEinstein condensates (BECs) [3-8] as well as in other areas such as quantum optics [9], disordered systems [10], and dynamical systems [11-13]. Nonetheless, while positive forms, characteristic of stable systems, are well known to be diagonalizable, i.e., they can be written in terms of normal coordinates and viewed as a set of independent bosons or separate oscillators (plus eventually free particle terms in the presence of standard zero-frequency modes [1,2]), nonpositive ones may not admit such a diagonal representation [14]. Nonpositive forms can arise in the description of BEC instabilities [6-8] and fast rotating condensates [15-20], as well as in generalized RPA treatments [21,22].

In [14] we have extended the standard methodology for diagonalizing quadratic bosonic forms by using generalized quasiparticle operators fulfilling bosonlike commutation relations, associated with non-Hermitian coordinates and momenta. This allows one to characterize the operators exhibiting purely exponential evolutions (complex modes) in nonpositive forms, enabling a precise description of the dynamics and quadratic invariants. We have also pointed out that nonpositive forms can in some cases be dynamically stable, as the evolution can remain quasiperiodic, irrespective of the initial conditions. Moreover, we have noticed the existence of nondiagonalizable cases where the equations of motion cannot be fully decoupled and which may arise even if all eigenfrequencies are nonzero. The method of Ref. [14] has been found useful in the context of BEC, being employed to study the emergence of instabilities in trapped BEC with a highly quantized vortex [6-8] through the Bogoliubov-de Gennes equations.

Here we will apply this methodology to the basic problem of a particle in a rotating anisotropic quadratic potential, not necessarily stable. This system is formally equivalent to that of a charged particle in a uniform magnetic field in a general quadratic potential $[15,16,23]$. The problem is therefore relevant for many fields. In particular, the rotating case has recently become relevant in the context of BEC in rotating anisotropic traps [15-20], which in the Landau-level approach are basically described by a cranked quadratic potential of the type considered here. The stable system is well known $[1,2,23,24]$ and diverse aspects of the stable anisotropic rotating case in the context of rotating condensates have recently been investigated [17-20].

We will examine here the general unstable case, which is of interest for fast rotating condensates as the Hamiltonian ceases to be positive definite at high frequency due to the centrifugal force. As we shall see, the unstable system exhibits a rich structure, with several different dynamical regimes as well as some quite remarkable features, including (a) the possibility of becoming nonseparable at the boundaries of regions with distinct dynamics, in the sense that the Hamiltonian can no longer be written as a sum of two independent standard or complex modes; in such cases the system will exhibit anomalous evolutions characterized by a set of linear equations which cannot be decoupled and which may lead to coordinates and/or momenta evolving with terms $\propto t e^{\lambda t}$ or even $\propto t^{3}$ and (b) the possibility of achieving dynamical stability in some unstable cases by increasing the magnetic field or tuning the rotational frequency. In particular, a stable anisotropic rotating potential becomes dynamically unstable just in a finite frequency window, recovering dynamical stability at high rotational frequency, whereas an unstable saddle-type potential can become dynamically stable in a certain frequency window.

In Sec. II we briefly revisit the main features of the formalism, discussing the concept of separability in generalized coordinates and momenta and the evolution for general nonseparable cases. The application is discussed in Sec. III while conclusions are drawn in Sec. IV.

\section{FORMALISM}

We consider a general quadratic Hamiltonian,

$$
h=\frac{1}{2} \sum_{i, j} T_{i j} p_{i} p_{j}+V_{i j} q_{i} q_{j}+U_{i j}\left(q_{i} p_{j}+p_{j} q_{i}\right),
$$




$$
=\frac{1}{2} R^{t} \mathcal{H}_{c} R, \quad R=\left(\begin{array}{l}
q \\
p
\end{array}\right), \quad \mathcal{H}_{c}=\left(\begin{array}{cc}
V & U \\
U^{t} & T
\end{array}\right),
$$

where $t$ denotes transpose, $T, V$ are symmetric matrices, and $p, q$ are Hermitian coordinates and momenta satisfying $\left[p_{i}, q_{j}\right]=-i \delta_{i j},\left[q_{i}, q_{j}\right]=\left[p_{i}, p_{j}\right]=0$, i.e.,

$$
R R^{t}-\left(R R^{t}\right)^{t}=\mathcal{M}_{c}, \quad \mathcal{M}_{c}=i\left(\begin{array}{cc}
0 & 1 \\
-1 & 0
\end{array}\right) .
$$

The ensuing Heisenberg equations of motion lead to a closed set of linear equations which can be written as

$$
\begin{gathered}
i \frac{d R}{d t}=-[h, R]=\widetilde{\mathcal{H}}_{c} R, \\
\tilde{\mathcal{H}}_{c}=\mathcal{M}_{c} \mathcal{H}=i\left(\begin{array}{cc}
U^{t} & T \\
-V & -U
\end{array}\right),
\end{gathered}
$$

where $\tilde{\mathcal{H}}_{c}$ represents the RPA matrix in coordinate representation [14]. It completely determines the system dynamics. Its eigenvalues come in pairs of opposite sign and can be complex in unstable systems. Moreover, $\widetilde{\mathcal{H}}_{c}$ can also be nondiagonalizable (as in the case of free particles $U=V=0, T_{i j}$ $=t_{i} \delta_{i j}$, although other cases can also arise, as discussed later). A positive definite $\mathcal{H}_{c}\left(R^{t} \mathcal{H}_{c} R>0 \forall\right.$ real $\left.R \neq 0\right)$ ensures a diagonalizable $\tilde{\mathcal{H}}_{c}$ together with a real spectrum $[1,2,14]$ (standard stable case), but the converse is not true.

Under a general linear canonical transformation $R=\mathcal{U} R^{\prime}$, with the matrix $\mathcal{U}$ satisfying $\mathcal{U} \mathcal{M}_{c} \mathcal{U}^{t}=\mathcal{M}_{c}$ in order to preserve Eq. (2), we have $\mathcal{H}_{c}^{\prime}=\mathcal{U}^{t} \mathcal{H}_{c} \mathcal{U}$ but $\tilde{\mathcal{H}}_{c}^{\prime}=\mathcal{M}_{c} \mathcal{H}_{c}^{\prime}$ $=\mathcal{U}^{-1} \tilde{\mathcal{H}}_{c} \mathcal{U}$, ensuring the invariance of the eigenvalues and the Jordan canonical form of $\widetilde{\mathcal{H}}_{c}$. Matrices $\widetilde{O} \equiv \mathcal{M}_{c} O$ are precisely those accounting for the closed algebra of the forms (1): if $O_{i}=\frac{1}{2} R^{t} \mathcal{O}_{i} R$, then

$$
\left[O_{i}, O_{j}\right]=\frac{1}{2} R^{t} \mathcal{C} R, \quad \text { with } \quad \widetilde{\mathcal{C}}=\left[\widetilde{\mathcal{O}}_{i}, \widetilde{\mathcal{O}}_{j}\right]
$$

It is obviously equivalent to using a representation of $h$ in terms of boson operators $b_{j}, b_{j}^{\dagger}=\left(q_{j} \pm i p_{j}\right) / \sqrt{2}$ satisfying $\left[b_{i}, b_{j}^{\dagger}\right]=\delta_{i j}$. Defining $Z=\left(\begin{array}{c}b \\ b^{\dagger}\end{array}\right)$ and the unitary matrix $\mathcal{S}$ $=\frac{1}{\sqrt{2}}\left(\begin{array}{cc}1 & i \\ 1 & -i\end{array}\right)$, such that $Z=\mathcal{S} R$, we may rewrite $h$ as

$$
\begin{gathered}
h=\sum_{i, j} A_{i j}\left(b_{i}^{\dagger} b_{j}+\frac{1}{2} \delta_{i j}\right)+\frac{1}{2}\left(B_{i j}^{+} b_{i}^{\dagger} b_{j}^{\dagger}+B_{i j}^{-} b_{i} b_{j}\right) \\
=\frac{1}{2} Z^{\dagger} \mathcal{H} Z, \quad \mathcal{H}=\mathcal{S} \mathcal{H}_{c^{\prime}} S^{\dagger}=\left(\begin{array}{cc}
A & B^{+} \\
B^{-} & A^{t}
\end{array}\right), \\
A=\frac{1}{2}\left[V+T-i\left(U-U^{t}\right)\right], \quad B^{ \pm}=\frac{1}{2}\left[V-T \pm i\left(U+U^{t}\right)\right] .
\end{gathered}
$$

The ensuing RPA matrix, defined by $i d Z / d t=\tilde{\mathcal{H}} Z$, is just $\tilde{\mathcal{H}}$ $=\mathcal{S} \tilde{\mathcal{H}}_{c} \mathcal{S}^{\dagger}$ and has obviously the same eigenvalues (and Jordan canonical form) as $\tilde{\mathcal{H}}_{c}$.

\section{A. General evolution and dynamical stability}

For a time-independent $h$, the solution of system (3) is

$$
R(t)=\exp \left[-i \widetilde{H}_{c} t\right] R,
$$

where $R \equiv R(0)$. Equation (7) is itself a linear canonical transformation. A system which is dynamically stable, i.e., leading to a bounded quasiperiodic evolution of all operators $p_{i}, q_{i}$, corresponds to a matrix $\widetilde{\mathcal{H}}_{c}$ which (i) is diagonalizable and (ii) has only real eigenvalues.

(a) If $\tilde{\mathcal{H}}_{c}$ is diagonalizable, such that $\tilde{\mathcal{H}}_{c}=\mathcal{W} \tilde{\mathcal{H}}_{c}^{\prime} \mathcal{W}^{-1}$ with $\left(\tilde{\mathcal{H}}_{c}^{\prime}\right)_{\mu \nu}=\lambda_{\nu} \delta_{\mu \nu}$, we may expand Eq. (7) as

$$
R(t)=\sum_{\nu} e^{-i \lambda_{\nu} t} \mathcal{W}_{\nu} Z_{\nu}^{\prime}
$$

where $\mathcal{W}_{\nu}$ is the $\nu$ th column of the eigenvector matrix $\mathcal{W}$ and $Z^{\prime}=\mathcal{W}^{-1} R$ is a set of normal operators satisfying

$$
i \frac{d Z_{\nu}^{\prime}}{d t}=\lambda_{\nu} Z_{\nu}^{\prime}
$$

and evolving then as $Z_{\nu}^{\prime}(t)=e^{-i \lambda_{\nu} t} Z_{\nu}^{\prime}$. For complex eigenvalues $\lambda_{\nu}$, these generalized normal operators represent exponentially increasing or decreasing modes (complex modes) and the dynamics is unbounded ( $\pm \lambda_{\nu}$ are both eigenvalues). They can always be ordered and normalized such that those associated with $\pm \lambda_{\nu}\left[b_{\nu}^{\prime}\right.$ and $\bar{b}_{\nu}^{\prime}$, with $\left.Z^{\prime}=\left(b^{\prime}, \bar{b}^{\prime}\right)^{t}\right]$ satisfy bosonlike commutation relations [14], i.e., $\left[b_{\nu}^{\prime}, \bar{b}_{\mu}^{\prime}\right]=\delta_{\mu \nu}$, $\left[b_{\mu}^{\prime}, b_{\nu}^{\prime}\right]=\left[\bar{b}_{\mu}^{\prime}, \bar{b}_{\nu}^{\prime}\right]=0$, but $\bar{b}_{\nu}^{\prime} \neq b_{\nu}^{\prime \dagger}$ if $\lambda_{\nu}$ is nonreal [14].

(b) If $\widetilde{\mathcal{H}}_{c}$ is nondiagonalizable, system (3) cannot be fully decoupled, but we may use its Jordan canonical form $\tilde{\mathcal{H}}_{c}$ $=\mathcal{W} \tilde{\mathcal{H}}_{c}^{\prime} \mathcal{W}^{-1}$, with $\tilde{\mathcal{H}}_{c}^{\prime}$ having blocks of the form

$$
\tilde{\mathcal{H}}_{c}^{\prime}=\left(\begin{array}{cccccccc}
\cdots & & & & & & \\
& \lambda_{\nu} & 1 & 0 & & 0 & \\
& 0 & \lambda_{\nu} & 1 & & 0 & \\
& & & & \ddots & & \\
& & & & & \lambda_{\nu} & \\
& & & & & & \ldots
\end{array}\right) .
$$

We may then expand Eq. (7) as

$$
R(t)=\sum_{\nu} e^{-i \lambda_{\nu} t} \sum_{k=1}^{d_{\nu}} \mathcal{W}_{\nu_{k}} \sum_{l=k}^{d_{\nu}} Z_{\nu_{l}}^{\prime} \frac{t^{l-k}}{(l-k) !},
$$

where again $Z^{\prime}=\mathcal{W}^{-1} R, d_{\nu}$ is the dimension of the block and $\nu_{k}\left(k=1, \ldots, d_{\nu}\right)$ labels elements within each block, with $\mathcal{W}_{\nu_{k}}$ as the $\nu_{k}$ column of the generalized eigenvector matrix $\mathcal{W}$. The generalized normal operators $Z$ ' satisfy the "minimally coupled" evolution equations allowed by the Jordan form, i.e., $i d Z^{\prime} / d t=\tilde{\mathcal{H}}_{c}^{\prime} Z^{\prime}$ or

$$
i \frac{d Z_{\nu_{k}}^{\prime}}{d t}=\lambda_{\nu} Z_{\nu_{k}}^{\prime}+\left(1-\delta_{k, d_{\nu}}\right) Z_{\nu_{k+1}}^{\prime},
$$


leading to $Z_{\nu_{k}}^{\prime}(t)=e^{-i \lambda_{\nu} t} \sum_{l=k}^{d_{\nu}} Z_{\nu_{l}}^{\prime} \frac{t^{l-k}}{(l-k) !}$. The dynamics is then unbounded even for real $\lambda_{\nu}$. The free particle case corresponds to $\lambda_{\nu}=0$ and $d_{\nu}=2$. Other cases are discussed in Sec. III.

\section{B. Separability}

We will denote Hamiltonian (1) as separable if there is a linear canonical transformation $R=\mathcal{U} R^{\prime}$ (with $\mathcal{U} \mathcal{M}_{c} \mathcal{U}^{t}=\mathcal{M}_{c}$ ) such that $\mathcal{H}_{c}^{\prime}=\mathcal{U}^{t} \mathcal{H}_{c} \mathcal{U}$ is diagonal. In this case we may then rewrite $h$ as a sum of independent elementary quadratic systems,

$$
h=\frac{1}{2} \sum_{\nu}\left(\alpha_{\nu} p_{\nu}^{\prime 2}+\beta_{\nu} q_{\nu}^{\prime 2}\right),
$$

where $\left[q_{\nu}^{\prime}, p_{\mu}^{\prime}\right]=i \delta_{\nu \mu}, \quad\left[q_{\nu}^{\prime}, q_{\mu}^{\prime}\right]=\left[p_{\nu}^{\prime}, p_{\mu}^{\prime}\right]=0$. The diagonal form (12) is not unique, as $p_{\nu}^{\prime}, q_{\nu}^{\prime}$ can be rescaled $\left[\left(p_{\nu}^{\prime}, q_{\nu}^{\prime}\right)\right.$ $\left.\rightarrow\left(\alpha p_{\nu}^{\prime}, q_{\nu}^{\prime} / \alpha\right)\right]$ or swapped $\left[\left(p_{\nu}^{\prime}, q_{\nu}^{\prime}\right) \rightarrow\left(q_{\nu}^{\prime},-p_{\nu}^{\prime}\right)\right]$, but the products $\alpha_{\nu} \beta_{\nu}=\lambda_{\nu}^{2}$ determine the eigenvalues of $\widetilde{\mathcal{H}}_{c}$ and are hence unique.

In contrast with the conventional normal-mode expansion of a positive definite $h$, each of the terms in Eq. (12) can here represent not only (i) a standard stable oscillator $\left(\alpha_{\nu}\right.$ $\left.>0, \beta_{\nu}>0\right)$, but also (ii) an "inverted" oscillator $\left(\alpha_{\nu}\right.$ $\left.<0, \beta_{\nu}<0\right)$, (iii) a generalized free particle $\left(\alpha_{\nu} \beta_{\nu}=0\right.$, with $\alpha_{\nu} \neq 0$ or $\beta_{\nu} \neq 0$, which can be standard or inverted), (iv) an "unstable oscillator" $\left(\alpha_{\nu} \beta_{\nu}<0\right)$, and (v) a "complex oscillator" $\left(\alpha_{\nu} \beta_{\nu}\right.$ complex), where $p_{\nu}^{\prime}, q_{\nu}^{\prime}$ are no longer Hermitian $\left(\mathcal{U}\right.$ complex). We should also add the vanishing case $(0) \alpha_{\nu}$ $=\beta_{\nu}=0$, where both $p_{\nu}^{\prime}$ and $q_{\nu}^{\prime}$ commute with $h$. Separability in Hermitian coordinates and momenta $(\mathcal{U}$ real $)$ is a restricted class of separability [14], since the eigenvalues $\pm \sqrt{\alpha_{\nu} \beta_{\nu}}$ of $\tilde{\mathcal{H}}_{c}$ are in such a case real or imaginary, while diagonalizable cases with full complex $\lambda_{\nu}$ do exist [14].

A diagonalizable $\tilde{\mathcal{H}}_{c}$ ensures separability since in this case we may rewrite $h$ in terms of the generalized normal operators $Z^{\prime}=\left(b^{\prime}, \bar{b}^{\prime}\right)^{t}$ of Eq. (9) as [14]

$$
h=\sum_{\nu} \lambda_{\nu}\left(\bar{b}_{\nu}^{\prime} b_{\nu}^{\prime}+\frac{1}{2}\right)=\frac{1}{2} \sum_{\nu} \lambda_{\nu}\left(p_{\nu}^{\prime 2}+q_{\nu}^{\prime 2}\right),
$$

where $q_{\nu}^{\prime}=\left(b_{\nu}^{\prime}+\bar{b}_{\nu}^{\prime}\right) / \sqrt{2}, p_{\nu}^{\prime}=\left(b_{\nu}^{\prime}-\bar{b}_{\nu}^{\prime}\right) /(\sqrt{2} i)$, i.e., $\mathcal{U}=\mathcal{W S}$. For complex $\lambda_{\nu}, \bar{b}_{\nu}^{\prime} \neq b_{\nu}^{\prime \dagger}$ and $p_{\nu}^{\prime}, q_{\nu}^{\prime}$ are non-Hermitian. In case (iv) we may still rewrite the ensuing term in Eq. (13) in terms of Hermitian $q_{\nu}^{\prime}, p_{\nu}^{\prime}$ by a complex rescaling $\left(p_{\nu}^{\prime}, q_{\nu}^{\prime}\right)$ $\rightarrow\left(p_{\nu}^{\prime} / \sqrt{i}, \sqrt{i} q_{\nu}^{\prime}\right)[14]$, while in (ii) we should choose $\lambda_{\nu}<0$ for $p_{\nu}^{\prime}, q_{\nu}^{\prime}$ Hermitian. Conversely, for $\alpha_{\nu} \beta_{\nu} \neq 0$ or $\alpha_{\nu}=\beta_{\nu}=0$, each term in Eq. (12) leads to a diagonalizable $2 \times 2$ block in $\tilde{\mathcal{H}}_{c}^{\prime}$. However, the separable case also includes the free particle case (iii) where $\tilde{\mathcal{H}}_{c}$ is nondiagonalizable, as $\tilde{\mathcal{H}}_{c}^{\prime}$ will contain a Jordan block $\widetilde{\mathcal{H}}_{0}^{\prime}=\left(\begin{array}{ll}0 & 1 \\ 0 & 0\end{array}\right)$ with $\lambda_{\nu}=0$. Here $\widetilde{\mathcal{H}}_{c}^{2}$ remains diagonalizable.

Hence, systems where $\tilde{\mathcal{H}}_{c}^{2}$ is nondiagonalizable, implying $\tilde{\mathcal{H}}_{c}^{\prime}$ having a Jordan block of dimension $d_{\nu}>2$ or $d_{\nu}=2$ and $\lambda_{\nu} \neq 0$, are nonseparable. They may arise even in simple unstable cases (Sec. III) and their evolution can be determined through the general solution (10).
Dynamically stable quadratic systems correspond to a separable $h$ with terms just of the form (i), (ii), or (0), and have then a discrete spectrum

$$
E_{\left\{n_{\nu}\right\}}=\sum_{\nu} \lambda_{\nu}\left(n_{\nu}+\frac{1}{2}\right),
$$

where $\lambda_{\nu}>0,<0$, or 0 in case (i), (ii), or (0). An example of a nonpositive dynamically stable form is an angular momentum component $l=q_{+} p_{-}-q_{-} p_{+}=\frac{1}{2}\left(p_{+}^{\prime 2}+q_{+}^{\prime 2}\right)-\frac{1}{2}\left(p_{-}^{\prime 2}+q_{-}^{\prime 2}\right)$, where $p_{ \pm}^{\prime}=p_{ \pm}-q_{\mp} / 2, q_{ \pm}^{\prime}=q_{ \pm} / 2+p_{\mp}^{\prime}$, which is the sum of a positive plus an inverted oscillator. Here $\widetilde{\mathcal{L}}_{c}$ is diagonalizable with eigenvalues $\pm 1, \mp 1$.

\section{APPLICATION}

We will consider the quantum problem of a particle in an anisotropic quadratic potential, not necessarily stable, rotating around one of its principal axes $(z)$. It is formally equivalent to that of a particle of charge $e$ in a uniform magnetic field $\boldsymbol{H}$ parallel to this axis in a quadratic potential. The Hamiltonian of the latter reads

$$
\begin{aligned}
H & =\frac{(\boldsymbol{P}-e \boldsymbol{A} / c)^{2}}{2 m}+\frac{1}{2}\left(K_{x} X^{2}+K_{y} Y^{2}+K_{z} Z^{2}\right), \\
& =\frac{1}{2}\left[\frac{P_{x}^{2}+P_{y}^{2}}{m}+K_{x}^{\prime} X^{2}+K_{y}^{\prime} Y^{2}-\Omega L_{z}\right]+H_{z},
\end{aligned}
$$

where $\boldsymbol{A}=\frac{1}{2} \boldsymbol{H} \times \boldsymbol{R}$ is the vector potential, $\Omega=e|\boldsymbol{H}| / m c$ is the cyclotron frequency, $L_{z}=X P_{y}-Y P_{x}$ is the angular momentum component, $H_{z}=\frac{1}{2}\left(\frac{P_{z}^{2}}{m}+K_{z} Z^{2}\right)$, and

$$
K_{x, y}^{\prime}=K_{x, y}+m \Omega^{2} / 4 \text {. }
$$

For $\Omega \rightarrow 2 \Omega$, Eq. (16) is just the cranked Hamiltonian describing the intrinsic motion of a particle in a rotating quadratic potential with constants $K_{x, y}^{\prime}$ : if $H(t)=U(t) H(0) U^{\dagger}(t)$, with $U(t)=e^{-i \Omega L_{z} t / \hbar}$, the Heisenberg equations for rotating operators $O(t)=U(t) O U^{\dagger}(t)$ are those for the $t$-independent cranked Hamiltonian $H=H(0)-\Omega L_{z}$.

Since $H_{z}$ is fully decoupled from the rest and its treatment is trivial, it will be omitted in what follows and all considerations will refer to the motion in the $x y$ plane. Defining dimensionless operators $\boldsymbol{q}=\boldsymbol{R} \sqrt{m \Omega_{0} / \hbar}, \boldsymbol{p}=\boldsymbol{P} / \sqrt{\hbar m \Omega_{0}}$ satisfying $\left[q_{\mu}, p_{\mu^{\prime}}\right]=i \delta_{\mu \mu^{\prime}}$, where $\Omega_{0}$ is a reference frequency, we can rewrite $h \equiv H_{x y} / \hbar \Omega_{0}$ as

$$
\begin{aligned}
h & =\frac{1}{2}\left[p_{x}^{2}+p_{y}^{2}+k_{x}^{\prime} q_{x}^{2}+k_{y}^{\prime} q_{y}^{2}\right]-\omega l_{z}, \\
& =\frac{1}{2} R^{t} \mathcal{H}_{c} R, \quad k_{x, y}^{\prime}=k_{x, y}+\omega^{2},
\end{aligned}
$$

with $k_{\mu}=K_{\mu} /\left(m \Omega_{0}^{2}\right), \omega=\Omega /\left(2 \Omega_{0}\right), \quad l_{z}=q_{x} p_{y}-q_{y} p_{x}$, and $R^{t}$ $=\left(q_{x}, q_{y}, p_{x}, p_{y}\right)$.

From the form of Eq. (15), it is apparent that for fixed $k_{\mu}$, the field cannot change the number of positive or negative eigenvalues of the Hamiltonian matrix $\mathcal{H}_{c}$ (the number of positive and negative diagonal elements is the same in any real diagonal representation of a quadratic form). For $m>0$, $\mathcal{H}_{c}$ will then have none, one, or two negative eigenvalues if 
and only if none, one, or both of the constants $k_{x}, k_{y}$ are, respectively, negative. The positive definite case corresponds then to $k_{x}>0, k_{y}>0 \forall \omega$, i.e., $k_{\mu}^{\prime}>\omega^{2}[17,18]$, although we will now see that dynamical stability is not restricted to this case.

\section{A. Dynamical stability}

The RPA matrix $\tilde{\mathcal{H}}_{c}=\mathcal{M}_{c} \mathcal{H}$ becomes

$$
\tilde{\mathcal{H}}_{c}=i\left(\begin{array}{cccc}
0 & \omega & 1 & 0 \\
-\omega & 0 & 0 & 1 \\
-k_{x}^{\prime} & 0 & 0 & \omega \\
0 & -k_{y}^{\prime} & -\omega & 0
\end{array}\right) \text {, }
$$

and its eigenvalues, which are the system eigenfrequencies (in units of $\hbar \Omega_{0}$ ), are $\pm \lambda_{+}, \pm \lambda_{-}$with

$$
\begin{gathered}
\lambda_{ \pm}=\sqrt{\left(k_{x}^{\prime}+k_{y}^{\prime}\right) / 2+\omega^{2} \pm \Delta}, \\
\Delta=\sqrt{\left(k_{x}^{\prime}-k_{y}^{\prime}\right)^{2} / 4+2 \omega^{2}\left(k_{x}^{\prime}+k_{y}^{\prime}\right)} .
\end{gathered}
$$

They satisfy $\lambda_{+}^{2} \lambda_{-}^{2}=\operatorname{det}\left[\mathcal{H}_{c}\right]=k_{x} k_{y}$. We first note that for $k_{x} k_{y} \neq 0$ and $\Delta \neq 0, \tilde{\mathcal{H}}_{c}$ is diagonalizable since it will have four different eigenvalues. We will then show that, for $\Delta$ $=0, \tilde{\mathcal{H}}_{c}$ is nondiagonalizable and, moreover, $h$ is nonseparable. At fixed $k_{\mu}, \Delta=0$ if $k_{x, y}<0$ and

$$
|\omega|=\omega_{c}^{ \pm}=\frac{1}{2}\left|\sqrt{-k_{x}} \pm \sqrt{-k_{y}}\right| .
$$

It also follows that $\lambda_{ \pm}$can be both real only if $k_{x} k_{y} \geq 0$. For a charged particle in a magnetic field, this opens up the possibility of full dynamical stability around a quadratic maximum $\left(k_{x, y}<0\right)$ but dismisses it for a saddle point $\left(k_{x} k_{y}<0\right)$. It is indeed verified that $\lambda_{ \pm}$are both real for $k_{x, y}>0$ as well as for $k_{x, y}<0$ and

$$
|\omega| \geq \omega_{c}^{+} .
$$

At fixed $k_{\mu}$, the dynamics in the vicinity of a quadratic maximum can then be stabilized by increasing the field. The behavior of $\lambda_{ \pm}$for increasing $|\omega|$ at fixed $k_{\mu}$ is depicted on the left panels of Fig. 1. As the scaled cyclotron frequency $2|\omega|$ is increased, at an anisotropic maximum, $\lambda_{ \pm}$evolve from imaginary $\left(|\omega| \leq \omega_{c}^{-}\right)$to full complex $\left(\omega_{c}^{-}<|\omega|<\omega_{c}^{+}\right)$and finally to real $\left(|\omega| \geq \omega_{c}^{+}\right)$values, reaching the system dynamical stability for $|\omega|>\omega_{c}^{+}$, whereas for $k_{x} k_{y}<0, \lambda_{+}$is real but $\lambda_{-}$is imaginary $\forall \omega$.

Stability in rotating potential. At fixed $k_{\mu}^{\prime}$, the previous picture is seen quite differently and leads to dynamical stability and instability windows in the anisotropic case $k_{x}^{\prime} \neq k_{y}^{\prime}$ (right panels in Fig. 1), i.e., when the rotation has a nontrivial effect. Owing to the centrifugal force, $\mathcal{H}_{c}$ is here positive definite just for $\omega^{2}<k_{x, y}^{\prime}$. However, $\lambda_{ \pm}$are real also for $\omega^{2}>k_{x, y}^{\prime}>0$, implying that motion in a rotating stable potential becomes dynamically stable at high rotational frequencies and dynamically unstable just in the finite interval

$$
\min \left[k_{x}^{\prime}, k_{y}^{\prime}\right] \leq \omega^{2} \leq \max \left[k_{x}^{\prime}, k_{y}^{\prime}\right],
$$

where $\lambda_{-}$becomes imaginary or 0 (see Sec. III B). In contrast, for a saddle point with $k_{x}^{\prime}>0>k_{y}^{\prime}$ (or vice versa) the

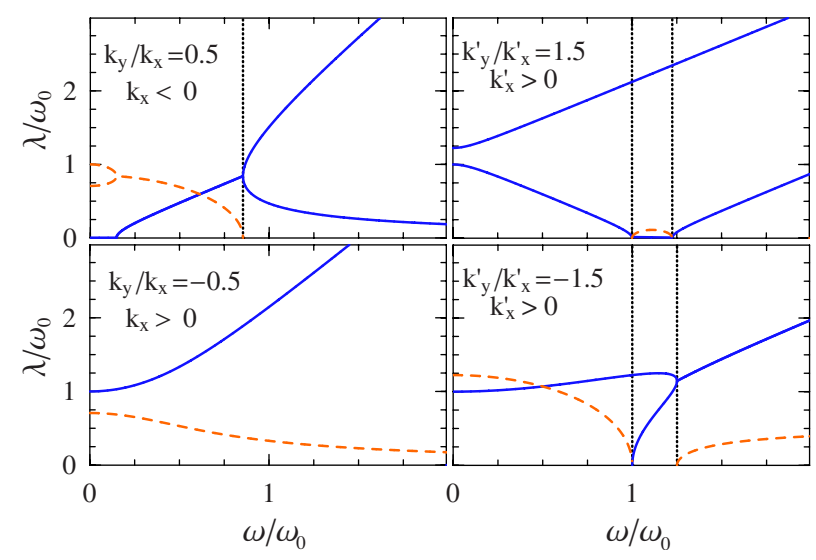

FIG. 1. (Color online) The real (solid lines) and imaginary (dashed lines) parts of eigenfrequencies (20) for selected fixed values of $k_{x, y}$ (left panels, corresponding to a particle in a magnetic field) and $k_{x, y}^{\prime}$ (right panels, corresponding to a particle in a rotating potential), in terms of the scaled cyclotron or rotational frequency. Vertical dotted lines separate dynamically stable and unstable regions. We have set $\omega_{0}=\sqrt{\left|k_{x}\right|}\left(\sqrt{k_{x}^{\prime}}\right)$ in the left (right) panels.

system becomes dynamically stable in the windows,

$$
\begin{gathered}
k_{x}^{\prime}<\omega^{2} \quad\left(-k_{x}^{\prime}<k_{y}^{\prime}<0\right), \\
k_{x}^{\prime}<\omega^{2}<\omega_{c}^{\prime 2} \quad\left(-3 k_{x}^{\prime}<k_{y}^{\prime}<-k_{x}^{\prime}\right),
\end{gathered}
$$

with $\omega_{c}^{\prime}=\frac{\left|k_{x}^{\prime}-k_{y}^{\prime}\right|}{\sqrt{-8\left(k_{x}^{\prime}+k_{y}^{\prime}\right)}}$ as the single value of $|\omega|$ where $\Delta=0$ at fixed $k_{\mu}^{\prime}$ (requires $k_{x}^{\prime}+k_{y}^{\prime}<0$ ). Thus, $l_{z}$ can turn unstable the dynamics of a stable anisotropic oscillator and stabilize that around a saddle point within limits (25). It can never stabilize a quadratic maximum.

In the isotropic case $k_{x}^{\prime}=k_{y}^{\prime}=k^{\prime},\left[h, l_{z}\right]=0$ and Eq. (20) leads then to $\left|\lambda_{ \pm}\right|=\left|\sqrt{k^{\prime}} \pm \omega\right|$. The rotation has no effect except for the shift $\pm \omega$. The instability window collapses into a single point $\omega^{2}=k^{\prime}$ where the dynamics remains stable (see below), since it corresponds to the Landau case $k_{x}=k_{y}=0$ $[23,25]$.

\section{B. Separability}

Let us now examine the separable representation of Eq. (17), feasible for $\Delta \neq 0$, and the ensuing distinct dynamical regimes. Defining, as in the stable case [1,2],

$$
p_{ \pm}=p_{x, y}+\gamma q_{y, x}, \quad q_{ \pm}=\frac{q_{x, y}-\eta p_{y, x}}{1+\gamma \eta}
$$

where $\quad \gamma=\frac{2 \Delta-k_{x}^{\prime}+k_{y}^{\prime}}{4 \omega}, \quad \eta=\frac{2 \gamma}{k_{x}^{\prime}+k_{y}^{\prime}} \quad$ and $\quad\left[q_{\nu}, p_{\mu}\right]=i \delta_{\mu \nu}, \quad\left[p_{\mu}, p_{\nu}\right]$ $=\left[q_{\mu}, q_{\nu}\right]=0$ for $\mu, \nu= \pm$ (with $\gamma, \eta \rightarrow 0$ if $\omega \rightarrow 0$ and $k_{x}^{\prime}$ $>k_{y}^{\prime}$ ), we may rewrite Eq. (17) for $\Delta \neq 0$ as a sum of two independent elementary quadratic forms,

$$
h=\frac{1}{2}\left(\alpha_{+} p_{+}^{2}+\beta_{+} q_{+}^{2}\right)+\frac{1}{2}\left(\alpha_{-} p_{-}^{2}+\beta_{-} q_{-}^{2}\right),
$$




$$
\alpha_{ \pm}=\frac{2 \Delta+k_{x}^{\prime}-k_{y}^{\prime} \pm 4 \omega^{2}}{4 \Delta}, \quad \beta_{ \pm}=\frac{\Delta\left(2 \Delta-k_{x}^{\prime}+k_{y}^{\prime} \pm 4 \omega^{2}\right)}{4 \omega^{2}},
$$

with $\alpha_{ \pm} \beta_{ \pm}=\lambda_{ \pm}^{2}$, For real $\Delta \neq 0, p_{ \pm}, q_{ \pm}$are Hermitian and $\lambda_{ \pm}$is real or imaginary. Equations (27) and (28) are, however, also applicable for imaginary $\Delta \neq 0$, where $p_{ \pm}, q_{ \pm}$are non-Hermitian and $\lambda_{ \pm}$complex. From the previously mentioned property of diagonal quadratic forms, it follows that all four coefficients $\alpha_{ \pm}, \beta_{ \pm}$must be positive for $k_{x, y}>0$, just one (two) of them will be negative for $k_{x} k_{y}<0\left(k_{x, y}<0\right.$ and $\Delta$ real), and one of them will vanish for $k_{y}=0, k_{x} \neq 0$ (or vice versa) whereas two of them will vanish in the Landau case $k_{x}=k_{y}=0$.

Diagonalizable cases. For $\Delta \neq 0$ and $k_{x} k_{y} \neq 0, \lambda_{ \pm} \neq 0$ and $\tilde{\mathcal{H}}_{c}$ is diagonalizable. Defining $p_{ \pm}^{\prime}=\sqrt{\alpha_{ \pm} / \lambda_{ \pm}} p_{ \pm}, \quad q_{ \pm}^{\prime}$ $=\sqrt{\beta_{ \pm} / \lambda_{ \pm}} q_{ \pm}$, we may rewrite Eq. (27) as

$$
h=\frac{1}{2} \sum_{\nu= \pm} \lambda_{\nu}\left(p_{\nu}^{\prime 2}+q_{\nu}^{\prime 2}\right)=\sum_{\nu= \pm} \lambda_{\nu}\left(\bar{b}_{\nu}^{\prime} b_{\nu}^{\prime}+\frac{1}{2}\right),
$$

where $b_{\nu}^{\prime}=\frac{q_{\nu}^{\prime}+i p_{\nu}^{\prime}}{\sqrt{2}}, \bar{b}_{\nu}^{\prime}=\frac{q_{\nu}^{\prime}-i p_{\nu}^{\prime}}{\sqrt{2}}$ are the generalized normal operators evolving as $b_{\nu}^{\prime}(t)=e^{-i \lambda_{\nu} t} b_{\nu}^{\prime}, \bar{b}_{\nu}^{\prime}(t)=e^{i \lambda_{\nu} t} \bar{b}_{\nu}^{\prime}$, which can be directly obtained from the eigenvectors of $\tilde{\mathcal{H}}_{c}$. At fixed $k_{\mu}$, the diagonalizable sectors are (Fig. 2)

(A) $k_{x, y}>0$ : here $\alpha_{ \pm}>0, \beta_{ \pm}>0$, with $\lambda_{ \pm}>0$. This is the positive definite case [case (i) in Sec. II B].

(B) $k_{x, y}<0,|\omega|>\omega_{c}^{+}$: here $\alpha_{+}>0, \beta_{+}>0$; but $\alpha_{-}<0, \beta_{-}$ $<0$, implying $\lambda_{+}>0$ but $\lambda_{-}<0$ for $p_{-}^{\prime}, q_{-}^{\prime}$ Hermitian. Equation (29) becomes a standard plus an inverted oscillator [cases (i)+(ii)], remaining dynamically stable.

(C) $k_{x, y}<0,|\omega|<\omega_{c}^{-}$: here $\alpha_{\nu} \beta_{\nu}<0$ for $\nu= \pm$ and $\lambda_{ \pm}$are both imaginary. Both terms in Eq. (27) are unstable oscillators [case (iv)], leading to $p_{ \pm}^{\prime}, q_{ \pm}^{\prime}$ non-Hermitian.

(D) $k_{x} k_{y}<0$ : here $\alpha_{+}>0, \beta_{+}>0$; but $\alpha_{-} \beta_{-}<0$, with $\lambda_{+}$ real and $\lambda_{-}$imaginary. Equation (12) becomes a stable plus an unstable oscillator $\left[\right.$ cases (i) $+($ iv) $]$, with $p_{-}^{\prime}, q_{-}^{\prime}$ nonHermitian.

In all previous cases $p_{ \pm}, q_{ \pm}$in Eq. (27) are Hermitian.

(E) $k_{x, y}<0, \omega_{c}^{-}<|\omega|<\omega_{c}^{+}$: here $\alpha_{ \pm}, \beta_{ \pm}$, and $\lambda_{ \pm}$are full complex and $p_{ \pm}, q_{ \pm}$as well as $p_{ \pm}^{\prime}, q_{ \pm}^{\prime}$ are non-Hermitian [case (v)]. They represent complex normal modes.

(F) $k_{x}=k_{y}=0$ (Landau case): here $\alpha_{+}=1, \beta_{+}=4 \omega^{2}$ whereas $\alpha_{-}=\beta_{-}=0$, leading to $\lambda_{+}=2|\omega|$ and $\lambda_{-}=0 . h$ is then a standard plus a vanishing oscillator [cases (i) +(0)]. This wellknown case $[23,25]$ is then dynamically stable in the $x y$ plane despite the vanishing eigenfrequency.

In cases $(\mathrm{A}),(\mathrm{B})$, and $(\mathrm{F}), p_{\nu}^{\prime}, q_{\nu}^{\prime}$ are Hermitian, with $\bar{b}_{\nu}^{\prime}$ $=b_{\nu}^{\prime \dagger}$, and $h$ possesses then a discrete spectrum,

$$
E_{n_{+}, n_{-}}=\lambda_{+} n_{+}+\lambda_{-} n_{-}+\frac{1}{2}\left(\lambda_{+}+\lambda_{-}\right),
$$

with $\lambda_{+}>0$, while $\lambda_{-}>0$ in case (A), $\lambda_{-}<0$ in case (B), and $\lambda_{-}=0$ in case $(\mathrm{F})$. These are the dynamically stable cases.

At fixed $k_{\mu}^{\prime}$ (rotating potential) all regions are just shifted by $+\omega^{2}$ (lower panel in Fig. 2). This shift leads to the different behavior of $\lambda_{ \pm}$with $\omega$ depicted in Fig. 1 .

Separable non-diagonalizable cases. They arise for $k_{y}$

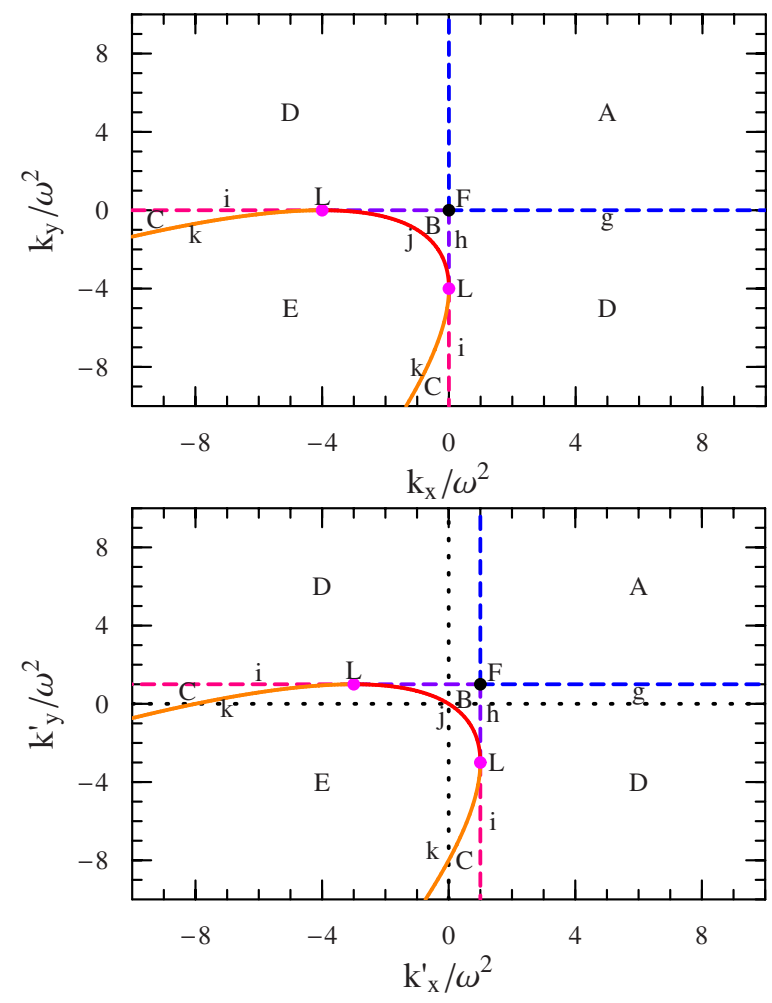

FIG. 2. (Color online) Top: regions in the $k_{x, y}$ plane at fixed $\omega$ with distinct dynamical regimes: $\mathrm{A}$ is the positive definite sector, $\mathrm{F}$ is the Landau point, B is the nonpositive dynamically stable sector, $\mathrm{C}$ and $\mathrm{D}$ are unstable regions with four and two imaginary eigenfrequencies, $\mathrm{E}$ is that with full complex eigenfrequencies, while the solid lines j,k depict the nonseparability curve $\Delta=0$, with degenerate real $(\mathrm{j})$ or imaginary $(\mathrm{k})$ eigenfrequencies (see text). The points $\mathrm{L}\left(k_{x}=0, k_{y}=-4 \omega^{2}\right.$ or vice versa) are the exceptional nonseparable cases with a single vanishing eigenfrequency. Dashed lines indicate separable cases with a free particle term (standard in $\mathrm{g}, \mathrm{i}$ and inverted in $\mathrm{h}$ ) and a stable $(\mathrm{g}, \mathrm{h})$ or an unstable (i) oscillator. Bottom: same details in the $k_{x, y}^{\prime}$ plane, corresponding to the rotating system; all regions and curves are just shifted by $\omega^{2}$. Plots in Fig. 1 depict the behavior of $\lambda_{ \pm}$along straight lines running from $\infty(\omega=0)$ to the origin $(|\omega| \rightarrow \infty)$.

$=0$ and $k_{x} \neq 0$ or vice versa (sectors g, h, and i in Fig. 2). For $k_{y}=0, \Delta=2 \omega^{2}+k_{x} / 2$ and we obtain $\lambda_{-}=0$ but $\lambda_{+}=\sqrt{4 \omega^{2}+k_{x}}$ $\neq 0$ for $\Delta \neq 0$, with

$$
h=\frac{1}{2}\left(p_{+}^{2}+\lambda_{+}^{2} q_{+}^{2}\right)+\frac{1}{2} \frac{k_{x}}{\lambda_{+}^{2}} p_{-}^{2} .
$$

In $\mathrm{g}, k_{x}>0$ and Eq. (31) corresponds to a stable oscillator $\left(\lambda_{+}>0\right)$ plus a free particle $[\operatorname{cases}(\mathrm{i})+(\mathrm{iii})]$. In $\mathrm{h},-4 \omega^{2}$ $<k_{x}<0$ and $\lambda_{+}$is still real, but the second term in Eq. (31) becomes negative: $h$ becomes a stable oscillator plus an inverted free particle term. The latter "absorbs" here the instability, allowing dynamical stability in the coordinates $p_{+}, q_{+}$. Finally, in sector i $\left(k_{x}<-4 \omega^{2}\right) \lambda_{+}$becomes imaginary: Eq. (31) corresponds here to an unstable oscillator plus a standard free particle [cases (iv) + (iii)]

Equation (31) leads to an evolution $\bar{b}_{+}^{\prime}(t)=e^{i \lambda_{+} t} \bar{b}_{+}^{\prime}, b_{+}^{\prime}(t)$ $=e^{-i \lambda_{+} t} b_{+}^{\prime}, \quad p_{-}(t)=p_{-}$, and $q_{-}(t)=\left(k_{x} / \lambda_{+}^{2}\right) p_{-} t+q_{-}$, which is 
characteristic of a nondiagonalizable $\tilde{\mathcal{H}}_{c}$ with a canonical form $\left(\mathcal{A} \sim \mathcal{B}\right.$ denotes $\left.\mathcal{A}=\mathcal{W}^{-1} \mathcal{B W}\right)$

$$
\widetilde{\mathcal{H}}_{c} \sim\left(\begin{array}{cccc}
\lambda_{+} & 0 & 0 & 0 \\
0 & -\lambda_{+} & 0 & 0 \\
0 & 0 & 0 & 1 \\
0 & 0 & 0 & 0
\end{array}\right) .
$$

\section{Nonseparability}

We now examine the very peculiar case $\Delta=0$ and $\omega \neq 0$, where the eigenfrequencies become degenerate, $\lambda_{ \pm}$ $=\sqrt{\frac{\left(3 k_{x}^{\prime}+k_{y}^{\prime}\right)\left(3 k_{y}^{\prime}+k_{x}^{\prime}\right)}{8\left(k_{x}^{\prime}+k_{y}^{\prime}\right)}}=\lambda$, and both $\widetilde{\mathcal{H}}_{c}$ and $\widetilde{\mathcal{H}}_{c}^{2}$ are nondiagonalizable. It occurs at the threshold for full complex solutions, i.e., $|\omega|=\omega_{c}^{ \pm}\left(\omega_{c}^{\prime}\right)$ at fixed $k_{\mu}\left(k_{\mu}^{\prime}\right)$ and corresponds at fixed $\omega$ to a parabola rotated $\pi / 4$ with respect to the $k_{x, y}$ axes, with vertex at $k_{x, y}=-\omega^{2}$, i.e., $k_{x, y}^{\prime}=0$ (curves $\mathrm{j}$ and $\mathrm{k}$ and point $\mathrm{L}$ in Fig. 2).

(j) Here $|\omega|=\omega_{c}^{+}$and $\lambda=\sqrt[4]{k_{x} k_{y}}=\sqrt{\omega_{c}^{+2}-\omega_{c}^{-2}}>0 . \widetilde{\mathcal{H}}_{c}$ can be reduced to two nontrivial Jordan blocks,

$$
\tilde{\mathcal{H}}_{c} \sim\left(\begin{array}{cccc}
\lambda & 1 & 0 & 0 \\
0 & \lambda & 0 & 0 \\
0 & 0 & -\lambda & 1 \\
0 & 0 & 0 & -\lambda
\end{array}\right),
$$

which indicates nonseparability. The transformation

$$
\begin{gathered}
p_{ \pm}=\frac{\sqrt{\omega_{c}^{+}}}{\sqrt[4]{-k_{x, y}}}\left(p_{x, y}+\omega_{c}^{-} q_{y, x}\right), \\
q_{ \pm}=\frac{\sqrt[4]{-k_{x, y}}}{\sqrt{\omega_{c}^{+}}} \frac{\omega_{c}^{+2}+\lambda^{2}}{2 \lambda^{2}}\left(q_{x, y}+\frac{\omega_{c}^{-}}{\omega_{c}^{+2}+\lambda^{2}} p_{y, x}\right)
\end{gathered}
$$

allows one to express $h$ in this case as

$$
h=\frac{1}{2}\left(p_{+}^{2}+p_{-}^{2}\right)-\lambda\left(q_{+} p_{-}-q_{-} p_{+}\right),
$$

which is the cranked Hamiltonian for a rotating free particle and is a basic nonseparable form: the equations of motion $d p_{ \pm} / d t= \pm \lambda p_{\mp}, d q_{ \pm} / d t= \pm \lambda q_{\mp}+p_{ \pm}$cannot be fully decoupled even though there is no vanishing eigenfrequency. More explicitly, defining the operators $b_{ \pm}^{\prime}=\frac{ \pm p_{+}+i p_{-}}{\sqrt{2}}, \bar{b}_{ \pm}^{\prime}=\frac{ \pm i q_{+}+q_{-}}{\sqrt{2}}$ satisfying $\left[b_{\nu}^{\prime}, \bar{b}_{\mu}^{\prime}\right]=\delta_{\mu \nu},\left[\bar{b}_{\nu}^{\prime}, \bar{b}_{\mu}^{\prime}\right]=\left[b_{\nu}^{\prime}, b_{\mu}^{\prime}\right]=0$, we can rewrite Eq. (36) as

$$
h=\lambda\left(\bar{b}_{+}^{\prime} b_{+}^{\prime}-\bar{b}_{-}^{\prime} b_{-}^{\prime}\right)-b_{+}^{\prime} b_{-}^{\prime} .
$$

The ensuing equations of motion, $i \frac{d b_{ \pm}^{\prime}}{d t}= \pm \lambda b_{ \pm}^{\prime}$, $i \frac{d \bar{b}_{ \pm}^{\prime}}{d t}$ $=\mp \lambda \bar{b}_{ \pm}^{\prime}+b_{\mp}^{\prime}$, correspond exactly to the Jordan form (33) and possess the general solution

$$
b_{ \pm}^{\prime}(t)=e^{\mp i \lambda t} b_{ \pm}^{\prime}, \quad \bar{b}_{ \pm}^{\prime}(t)=e^{ \pm i \lambda t}\left(\bar{b}_{ \pm}^{\prime}-i t b_{\mp}^{\prime}\right),
$$

which gives rise to unbounded spiral-like trajectories in the variables $p_{ \pm}, q_{ \pm}$. This is apparent from Eq. (36): since $[l, h]=0$, where $l=q_{+} p_{-}-q_{-} p_{+}$, the evolution operator $e^{-i h t}$ $=e^{i \lambda l t} e^{-i(h+\lambda l) t}$ represents a rotation of frequency $\lambda$ applied to a free motion. Such motion cannot arise from a separable $h$. Although $h$ is the sum of two commuting quadratic forms and the equations of motion can be reduced to two separate blocks, the operators in each block do not commute with those of the other.

(k) Here $|\omega|=\omega_{c}^{-}$and $\lambda=i \sqrt[4]{k_{x} k_{y}}=\sqrt{\omega_{c}^{-2}-\omega_{c}^{+2}} \neq 0$ is imaginary. This case arises for $k_{x} \neq k_{y}$ and leads to the same canonical form (33). Replacing $\omega_{c}^{+} \leftrightarrow \omega_{c}^{-}$in Eqs. (34) and (35) leads here to

$$
h=\frac{1}{2}\left(p_{+}^{2}-p_{-}^{2}\right)-|\lambda|\left(q_{+} p_{-}+q_{-} p_{+}\right),
$$

where $p_{ \pm}, q_{ \pm}$are Hermitian. With a complex scaling $p_{-}$ $\rightarrow i p_{-}, q_{-} \rightarrow-i q_{-}$Eq. (38) becomes identical to Eq. (36) with an imaginary $\lambda$. The remaining equations remain then unchanged but lead to exponentially increasing or decreasing evolutions for the operators $b_{ \pm}^{\prime}, \bar{b}_{ \pm}^{\prime}$, corresponding to a "boost" $e^{-|\lambda| l t}$ applied to a free motion.

(L) This is an exceptional critical point where cases (B), (C), (D), (E), (h), (i), (j), and (k) merge. Here $k_{y}=0, k_{x}$ $=-4 \omega^{2}$ (i.e., $k_{y}^{\prime}=\omega^{2}, k_{x}^{\prime}=-3 \omega^{2}$ ) or vice versa, implying $|\omega|$ $=\omega_{c}^{+}=\omega_{c}^{-}$and $\lambda_{ \pm}=0$ : all four eigenvalues of $\tilde{\mathcal{H}}_{c}$ vanish. Nonetheless, $\widetilde{\mathcal{H}}_{c}$ is still of rank 3 , implying that it becomes similar to a full Jordan block $\left(d_{\nu}=4\right)$,

$$
\tilde{\mathcal{H}}_{c} \sim\left(\begin{array}{cccc}
0 & 1 & 0 & 0 \\
0 & 0 & 1 & 0 \\
0 & 0 & 0 & 1 \\
0 & 0 & 0 & 0
\end{array}\right)
$$

instead of two free particle blocks, as would appear from Eq. (33) for $\lambda \rightarrow 0$. In this case the equations of motion cannot be even partially decoupled. The transformation

$$
p_{ \pm}=\delta_{ \pm}\left(p_{x, y}+\omega q_{y, x}\right), \quad q_{ \pm}=\frac{1}{4 \delta_{ \pm}}\left(3 q_{x, y}-\omega^{-1} p_{y, x}\right)
$$

with $\delta_{+}=1, \delta_{-}=2$, allows one to rewrite $h$ at this point as

$$
h=\frac{1}{2} p_{+}^{2}-\omega q_{+} p_{-},
$$

which is again a basic nonseparable form: the ensuing equations of motion, $\frac{d p_{-}}{d t}=0, \frac{d p_{+}}{d t}=\omega p_{-}, \frac{d q_{+}}{d t}=p_{+}, \frac{d q_{-}}{d t}=-\omega q_{+}$, exhibit the structure of the Jordan form (39) for $\omega \neq 0$ and lead to a polynomial evolution of third degree in $t$ for $q_{-}$,

$$
\begin{gathered}
p_{-}(t)=p_{-}, \quad p_{+}(t)=p_{+}+\omega t p_{-}, \\
q_{+}(t)=q_{+}+p_{+} t+\frac{1}{2} \omega t^{2} p_{-}, \\
q_{-}(t)=q_{-}-\omega t q_{+}-\frac{1}{2} \omega t^{2} p_{+}-\frac{1}{6} \omega^{2} t^{3} p_{-} .
\end{gathered}
$$

Coordinates $q_{+}, q_{-}$experience then a constant and linearly increasing acceleration, respectively. In terms of the operators $\bar{b}_{-}^{\prime}=-\omega p_{-}, b_{+}^{\prime}=i p_{+}, \bar{b}_{+}^{\prime}=q_{+}$, and $b_{-}^{\prime}=i q_{-} / \omega$, which satisfy $\left[b_{\nu}^{\prime}, \bar{b}_{\mu}^{\prime}\right]=\delta_{\mu \nu},\left[b_{\mu}^{\prime}, b_{\nu}\right]=\left[\bar{b}_{\mu}^{\prime}, \bar{b}_{\nu}^{\prime}\right]=0$, we may also express Eq. (41) as 


$$
h=\bar{b}_{+}^{\prime} \bar{b}_{-}^{\prime}-\frac{1}{2} b_{+}^{\prime 2} .
$$

Their equations of motion, $d \bar{b}_{-}^{\prime} / d t=0, i d b_{+}^{\prime} / d t=\bar{b}_{-}^{\prime}, i d \bar{b}_{+}^{\prime} / d t$ $=b_{+}^{\prime}, i d b_{-}^{\prime} / d t=\bar{b}_{+}^{\prime}$, follow exactly the Jordan form (39). Note that the disappearance of one of the kinetic terms in Eq. (41) is not exceptional for a nonseparable form: Eq. (36) [and hence Eq. (38)] can also be rewritten as $p_{+}^{2}-\lambda l$ if $q_{ \pm} \rightarrow q_{ \pm}$ $+\frac{1}{2 \lambda} p_{\mp}$. We may also rewrite Eq. (41) with two kinetic terms with a similar transformation.

Equation (39) suggests that this case could be considered as a "free inseparable pair," generalizing the free particle case where $\widetilde{\mathcal{H}}_{c} \sim\left(\begin{array}{ll}0 & 1 \\ 0 & 0\end{array}\right)$. For a free particle $\tilde{\mathcal{H}}_{c}^{2}=0$ while here $\tilde{\mathcal{H}}_{c}^{4}=0$.

\section{CONCLUSIONS}

We have first analyzed, within the formalism of Ref. [14], the dynamics in general unstable quadratic bosonic forms, discussing the treatment of the general nondiagonalizable case and determining the conditions for dynamical stability and separability. We have then applied the formalism to the basic problem of a particle in a general rotating quadratic potential, relevant in the context of fast rotating condensates in harmonic traps and formally equivalent to that of a charged particle in a uniform magnetic field in a quadratic potential. The present analysis unveils the rich variety of behaviors that can be exhibited by the unstable system, sum- marized in Fig. 2, together with some quite remarkable features, which could lead to observable effects in fast rotating condensates. In particular, we have determined (a) the regions of dynamical stability; intrinsic motion in a rotating stable potential remains dynamically stable at high frequencies, becoming unstable just in a finite frequency window in the anisotropic case, whereas in a rotating saddle potential it can become dynamically stable in a certain window [Eqs. (24) and (25) and Fig. 1]; (b) the regions in parameter space where $H_{x y}$ can be written as a sum of two independent quadratic systems (separability), employing non-Hermitian normal coordinates and momenta if necessary (sector E), and those where such a representation is not feasible (nonseparability); (c) the explicit transformations and final forms for all cases, including the energy spectrum in the dynamically stable cases and the minimally coupled standard forms and equations of motion in the nonseparable cases; and (d) the existence of an exceptional nonseparable zero mode case (point L in Fig. 2) where all eigenfrequencies vanish and the Jordan block has a dimension of 4 . It is not equivalent to a standard zero-frequency mode and leads to coordinates evolving as a third degree polynomial in time. These results indicate that similar peculiar effects can arise in more complex unstable quadratic systems, which can be analyzed with the same general formalism and techniques of Sec. II.

\section{ACKNOWLEDGMENT}

R.R. and A.M.K. are supported by CIC of Argentina.
[1] P. Ring and P. Schuck, The Nuclear Many-Body Problem (Springer, New York, 1980).

[2] J. P. Blaizot and G. Ripka, Quantum Theory of Finite Systems (MIT Press, Cambridge, MA, 1986).

[3] E. V. Goldstein and P. Meystre, Phys. Rev. A 55, 2935 (1997).

[4] H. Pu and N. P. Bigelow, Phys. Rev. Lett. 80, 1134 (1998); C. K. Law, H. Pu, N. P. Bigelow, and J. H. Eberly, ibid. 79, 3105 (1997).

[5] S. Alexandrov and V. V. Kavanov, J. Phys.: Condens. Matter 14, L327 (2002); V. I. Yukalov and E. P. Yukalova, Laser Phys. Lett. 1, 50 (2004).

[6] E. Fukuyama, M. Mine, M. Okumura, T. Sunaga, and Y. Yamanaka, Phys. Rev. A 76, 043608 (2007); M. Mine et al., Ann. Phys. (N.Y.) 322, 2327 (2007).

[7] T. Sunaga et al., J. Low Temp. Phys. 148, 381 (2007); M. Mine et al., ibid. 148, 331 (2007).

[8] Y. Nakamura, M. Mine, M. Okumura, and Y. Yamanaka, Phys. Rev. A 77, 043601 (2008).

[9] P. Meystre and M. Sargent, Elements of Quantum Optics (Springer, New York, 1991).

[10] V. Gurarie and J. T. Chalker, Phys. Rev. Lett. 89, 136801 (2002).

[11] I. A. Pedrosa, Phys. Rev. A 55, 3219 (1997).
[12] V. V. Dodonov, J. Phys. A 33, 7721 (2000).

[13] A. M. Kowalski et al., Phys. Lett. A 297, 162 (2002).

[14] R. Rossignoli and A. M. Kowalski, Phys. Rev. A 72, 032101 (2005).

[15] A. Aftalion, X. Blanc, and J. Dalibard, Phys. Rev. A 71 , 023611 (2005); S. Stock et al., Laser Phys. Lett. 2, 275 (2005).

[16] I. Bloch, J. Dalibard, and W. Zwerger, Rev. Mod. Phys. 80, 885 (2008).

[17] M. Linn, M. Niemeyer, and A. L. Fetter, Phys. Rev. A 64, 023602 (2001).

[18] A. L. Fetter, Phys. Rev. A 75, 013620 (2007).

[19] M. Ö. Oktel, Phys. Rev. A 69, 023618 (2004).

[20] A. Aftalion, X. Blanc, and N. Lerner, Phys. Rev. A 79, 011603(R) (2009).

[21] H. Attias and Y. Alhassid, Nucl. Phys. A. 625, 565 (1997).

[22] R. Rossignoli and N. Canosa, Phys. Lett. B 394, 242 (1997); R. Rossignoli, N. Canosa, and P. Ring, Phys. Rev. Lett. 80, 1853 (1998); Phys. Rev. B 67, 144517 (2003).

[23] A. Feldman and A. H. Kahn, Phys. Rev. B 1, 4584 (1970).

[24] J. G. Valatin, Proc. R. Soc. London, Ser. A 238, 132 (1956).

[25] L. D. Landau, Z. Phys. 64, 629 (1930). 